\title{
High Accuracy Gamma Function Values for Some Rational Arguments
}

\author{
By D. C. Galant and P. F. Byrd
}

During the course of determining accurate Gaussian quadrature formulas for certain nonclassical weight functions, it was necessary to compute $\Gamma(p / q)$ to $60 \mathrm{D}$ to obtain moments to high accuracy. In particular, we needed $\Gamma(p / q)$ for $p=1,2, \cdots, q-1,2 p \neq q$, and $q=3,4,5,8,10$. The quantities $\Gamma(p / q)$, which for special cases also arise in the computation of Bessel functions of fractional order or of the Airy functions, were calculated using an interpretive routine that treated floating-point numbers of 70 significant figures.

Table 1

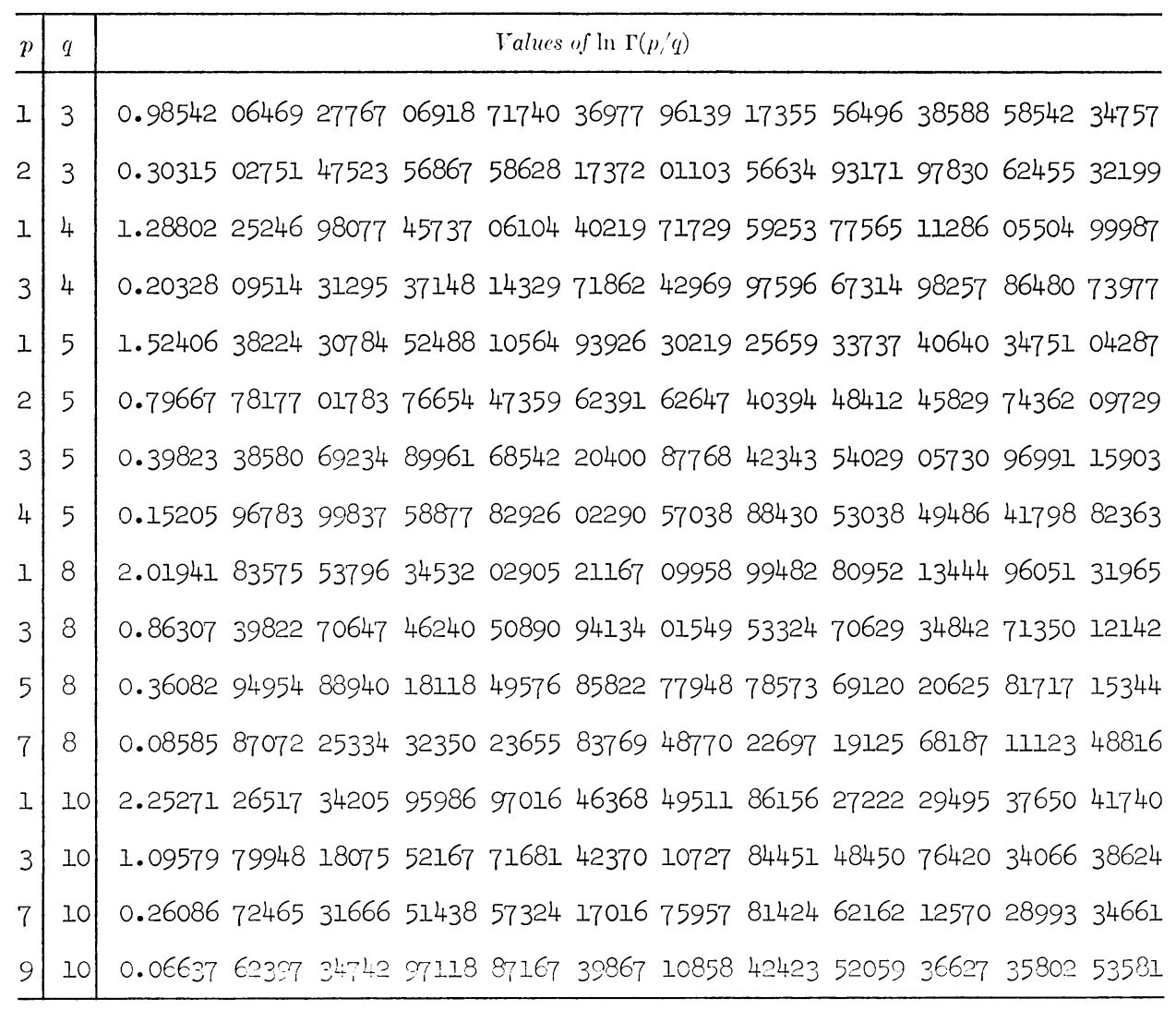

As in Sherry and Fulda [1], use is made of the asymptotic expansion

Received March 24, 1968. 
(1) $\ln \Gamma(p / q) \sim L(p / q, n)+\sum_{r=1}^{\infty} C_{r} /(n+p / q)^{2 r-1} \quad$ (n a positive integer) where $C_{r}=B_{2 r} / 2 r(2 r-1), B_{2 r}$ are Bernoulli numbers, and

$$
\begin{aligned}
L(p / q, n)= & (n-1 / 2+p / q) \ln (n q+p)-(n+p / q) \\
& +1 / 2 \ln (2 \pi)+(1 / 2-p / q) \ln q-\sum_{j=0}^{n-1} \ln (p+j q) .
\end{aligned}
$$

The function $\Gamma(x)$ is then calculated from

$$
\Gamma(x)=\exp (0.1 m) \exp [\ln \Gamma(x)-0.1 m],
$$

where $m$ is taken to be the greatest integer in $10 \ln \Gamma(x)$. Mansell's tables [2] provided the values of $(1 / 2) \ln (2 \pi)$ and logarithms of integers; the numbers $B_{k}$ are found in Davis [3].

With the above procedure, we obtained Tables 1 and 2 giving values of ln $\Gamma(p / q)$ and $\Gamma(p / q)$ to 60 decimal places.

TABLE 2

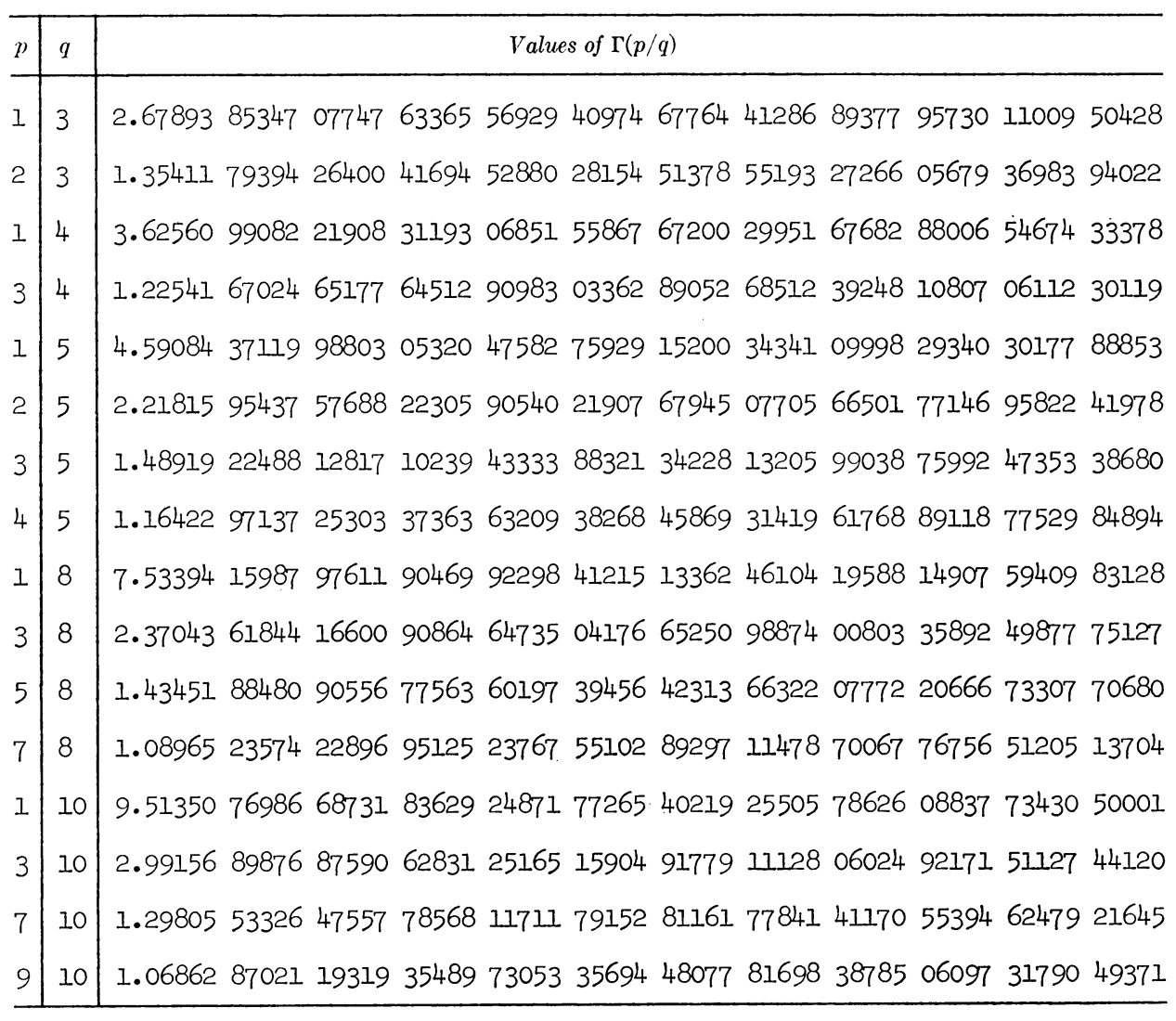

The calculated values were checked by means of the identity

$$
\Gamma(x) \Gamma(1-x)=\pi / \sin (\pi x) .
$$


In all cases, the two sides agreed to 64 decimal places. Also, the values of $\Gamma(1 / 3)$, $\Gamma(2 / 3), \ln \Gamma(1 / 3)$, and $\ln \Gamma(2 / 3)$ agree with the $35 \mathrm{D}$ calculations of Sherry and Fulda [1].

National Aeronautics and Space Administration

Ames Research Center

Moffett Field, California 94035

1. M. E. SHERRY \& S. FULDA, "Calculation of gamma functions to high accuracy," Math. Comp., v. 13, 1959, pp. 314-315. MR 21 \#7603.

2. W. E. Mansell, Tables of Natural and Common Logarithms to 110 Decimals, Roy. Soc. Math. Tables, v. 8, Cambridge Univ. Press, New York, 1964. MR 29 \#3675.

3. H. T. Davis, Tables of the Higher Mathematical Functions. Vol. II, Principia Press of Trinity University, San Antonio, Texas, 1935; rev. ed., 1963. 